\title{
Safety and effectiveness of endoluminal vacuum-assisted closure for esophageal defects: Systematic review and meta-analysis
}

다(1)우우

\author{
Authors \\ Muhammad Aziz' ${ }^{1}$, Hossein Haghbin², Sachit Sharma ${ }^{3}$, Simcha Weissman ${ }^{4}$, Saad Saleem5, Wade Lee-Smith ${ }^{6}$, Abdallah \\ Kobeissy ${ }^{1}$, Ali Nawras ${ }^{1}$, Yaseen Alastal ${ }^{1}$
}

Institutions

1 Division of Gastroenterology and Hepatology, University of Toledo, Toledo, Ohio, United States

2 Department of Internal Medicine, University of Toledo, Toledo, Ohio, United States

3 Department of Internal Medicine, Promedica Toledo Hospital, Toledo, Ohio, United States

4 Department of Internal Medicine, Hackensack Meridian Health Palisades Medical Center, North Bergen, New Jersey, United States

5 Department of Internal Medicine, Sunrise Hospital and Medical center, Las Vegas, Nevada, United States

6 Mulford Health Sciences Library, University of Toledo, Toledo, Ohio, United States

submitted 25.1.2021

accepted after revision $\quad 5.5 .2021$

Bibliography

Endosc Int Open 2021; 09: E1371-E1380

DOI 10.1055/a-1508-5947

ISSN 2364-3722

(c) 2021. The Author(s).

This is an open access article published by Thieme under the terms of the Creative Commons Attribution-NonDerivative-NonCommercial License, permitting copying and reproduction so long as the original work is given appropriate credit. Contents may not be used for commercial purposes, or adapted, remixed, transformed or built upon. (https://creativecommons.org/licenses/by-nc-nd/4.0/)

Georg Thieme Verlag KG, Rüdigerstraße 14,

70469 Stuttgart, Germany

Corresponding author

Muhammad Aziz, MD, University of Toledo Medical Center, Toledo, Ohio, United States

marajani@hotmail.com
\# Supplementary material is available under

https://doi.org/10.1055/a-1508-5947

\section{ABSTRACT}

Background and study aims Esophageal defects (leaks, fistulas, and perforations) are associated with significant morbidity and mortality. Endoluminal vacuum-assisted closure (EVAC) is a novel intervention that entails the use of sponges in the defect along with negative pressure to achieve granulation tissue formation and healing and has been gaining popularity. We performed a systematic review and pooled analysis of available literature to assess the safety and effectiveness of EVAC for esophageal defects.

Patients and methods We queried PubMed/Medline, Embase, Cochrane, and Web of Science through September 25, 2020 to include all pertinent articles highlighting the safety and effectiveness profile of EVAC for esophageal defects. Pooled rates, $95 \%$ confidence intervals (Cls), and heterogeneity $\left(R^{2}\right)$ were assessed for each outcome.

Results A total of 18 studies with 423 patients were included (mean age 64.3 years and males $74.4 \%$ ). The technical success for EVAC was $97.1 \%\left(\mathrm{Cl}: 95.4 \%-98.7 \%, I^{2}=0 \%\right)$. The clinical success was $89.4 \%\left(\mathrm{Cl}: 85.6 \%-93.1 \%, l^{2}=36.8 \%\right)$. The overall all-cause mortality and adverse events (AEs) noted were $7.1 \%\left(\mathrm{Cl}: 4.7 \%-9.5 \%, \mathrm{P}^{2}=0 \%\right)$ and $13.6 \%(\mathrm{Cl}: 8.0 \%-$ $19.1 \%, R^{2}=68.9 \%$ ), respectively. The pooled need for adjuvant therapy was $15.7 \%\left(\mathrm{Cl}: 9.8 \%-21.6 \%, \mathrm{I}^{2}=71.1 \%\right)$.

Conclusions This systematic review and meta-analysis showed high rates of technical success, clinical success, and low all-cause mortality and AEs using EVAC. Although the technique is a promising alternative, the lack of comparative studies poses a challenge in making definite conclusions regarding use of EVAC compared to other endoscopic modalities, such as clips and stents. 


\section{Introduction}

Esophageal defects (leaks, fistula, and perforation) are complications associated with esophageal procedures such as endoscopy, dilation, transesophageal echocardiography, variceal therapy, nasogastric tube placement, surgeries such as esophagectomy, trauma, malignancy, and ingestion [1-3]. Esophageal defects can also be spontaneous without the presence of external factors, as mentioned above [2]. Esophageal perforations, although rare, have a mortality rate ranging from $10 \%$ to $40 \%$ [4]. Esophageal leaks are also associated with increased mortality in patients (odds ratio [OR]: 3.00, $95 \%$ confidence interval (CI) 1.2-7.2) compared to having no leaks [3].

There are multiple ways to address these aforementioned defects. Historically, the only available modalities were a conservative approach or surgery [5]. For many patients, the traditional modalities lacked safety and effectiveness. With the development of biomaterials in the last two decades, endoscopic placement of self-expanding metal stent (SEMS) became the treatment of choice for less severe esophageal intrathoracic leaks [6]. SEMS was suitable for both nonsurgical and surgical candidates and showed superior effectiveness and a better safety profile than surgery or a conservative approach, when indicated [7]. Bleeding, stent migration, and perforation are adverse events (AES) associated with SEMS that can be fatal; therefore, there has been a search for better modalities [8]. A promising emerging alternative is endoluminal vacuum-assisted closure (EVAC). EVAC consists of placement of a sponge in the defect cavity or the esophageal lumen via the endoscope and connection of it to a negative pressure continuous vacuum [9]. EVAC treats the defect via mechanisms of leak source control, granulation tissue formation, encouraging reperfusion, and debridement of necrotic tissue once sponges are removed or replaced $[9,10]$. Modified from skin wound vacuum therapy, EVAC was introduced a decade ago for lower rectal anastomosis leaks and recently has gained popularity as an alternative treatment for defects in the upper gastrointestinal tract [11].

Literature is lacking regarding the overall effectiveness of outcomes related to the use of EVAC therapy for esophageal defects. We conducted a systematic review and pooled analysis of the available literature to assess the safety and effectiveness of EVAC for esophageal defects.

\section{Methods}

We performed this systematic review and meta-analysis using the framework laid out in "Preferred reporting items for systematic reviews and meta-analyses (PRISMA)" and "Meta-analyses Of Observational Studies in Epidemiology (MOOSE)" checklist $[12,13]$.

\section{Literature search/strategy}

The literature search was conducted in consultation with an experienced librarian (W.L.S). We queried the following databases from inception through September 25th, 2020: PubMed/Medline, Embase, Cochrane Register of Controlled Trials, and Web of Science Core Collection. Controlled subject terms and key- word synonyms for the concepts of "esophageal", "leak", "Fistula", "perforation", and "endoscopic vacuum therapy" were formulated for search on PubMed/Medline and translated to syntax / vocabulary of other databases. The search strategy created by the librarian (W.L.S) was cross checked by another reviewer (M.A.). Screening of studies was initially conducted based on titles and abstracts. Full text of relevant articles was further screened for interventions and outcomes of interests. Two independent reviewers (M.A. and H.H.) independently performed screening. Discrepancies were resolved through mutual discussion. A bibliography of the finalized articles was further hand screened to broaden the literature search and relevant articles were included. Details of the search strategy using EMBASE are highlighted in Supplementary Table 1.

\section{Study definitions}

Leaks are defined as a disruption of anastomosis resulting in fluid collection. Perforation refers to a full-thickness defect of the gastrointestinal tract. Fistula is an abnormal connection between two epithelialized surfaces. Although different, studies in the literature have used these terms interchangeably [14]. For simplicity, we will refer to these as "defects." The term nonsurgical iatrogenic refers to perforation caused by a diagnostic and/or therapeutic modality other than surgery on the esophagus. Technical success refers to the successful use of EVAC without technical failure. Clinical success refers to the successful use of EVAC therapy to achieve a clinical endpoint, that is, closure of the defect. Overall mortality is defined as mortality from any cause and not limited to intervention. AEs are defined as complications from the use of EVAC, such as stenosis, bleeding, dislocation of sponge, and visceral injury during the procedure.

\section{Inclusion and exclusion criteria}

Studies were included based on the following:

1. inclusion of patients with esophageal defects;

2. EVAC as an intervention;

3. control not applicable; and

4. outcomes of technical success, clinical success, all-cause mortality, treatment duration, hospital length of stay, and overall AEs.

We only included randomized controlled trials (RCTs), cohort studies (retrospective/prospective), and case control studies. Single-arm studies as well as an EVAC arm in comparative studies were included for this systematic review. We excluded other study designs, such as editorials, case reports, and case series with $<10$ patients, review articles, and meta-analyses. We excluded abstracts as they provide limited information in terms of overall study quality. We did not restrict our search to language. If more than one study was available from a particular center, we used the information from the most recent and updated one. 


\section{Data collection}

Data collection was performed by two independent reviewers (M.A. and H.H.). Any conflicts were resolved through mutual discussion. We extracted the following pertinent data: demographics (age, gender), cause of esophageal leak (nonsurgical iatrogenic, postsurgical, or spontaneous), use of adjuvant modalities/therapy (surgery, clips, stents, etc.) and outcomes (as above). The data were extracted directly in Microsoft Excel (Microsoft, Redmond, Washington, United States).

\section{Data synthesis and analysis}

The analysis was conducted using Open Meta Analyst (CEBM, University of Oxford, Oxford, United Kingdom) and Comprehensive Meta-Analysis (Biostat, Englewood, USA). Weighted random effects models were used to calculate proportional estimates of outcomes using DerSimonian-Laird method. Pooled outcomes with $95 \% \mathrm{Cls}$ were calculated and presented. A correction factor of 0.5 was applied if the event rate for a study outcome was 0 . For each outcome, $95 \%$ prediction interval ( $\mathrm{PI})$ was also generated [15]. Given the presumed heterogeneity of study population, random effects model was chosen over fixed effect model. The fixed effect model was used as a sensitivity tool. 12 statistics was used to calculate heterogeneity between studies with values of $0 \%, 25 \%, 50 \%$, and $75 \%$ interpreted as absent, low, moderate, and high heterogeneity, respectively [16].

\section{Subgroup analysis}

Subgroup analysis was performed for studies with sample size $\geq$ 20 patients and for studies published from 2015 on.

\section{Bias assessment}

Study quality was assessed using the Newcastle Ottawa score for cohort studies [17]. Publication bias was assessed using funnel plots (for qualitative assessment) and Begg's rank correlation test (for quantitative assessment using $P$ value).

\section{Results}

A total of 18 studies were included in the final analysis after applying the strict inclusion and exclusion criteria (supplementary material). Details of the study selection process are shown in - Fig. 1. A total number of 423 patients were included in these studies (mean age 64.3 years, $\mathrm{Cl}$ 62.7-65.9 years, and males $74.4 \%, \mathrm{Cl} 64.6 \%-84.3 \%$ ). The studies were published between 2010 and 2020. Baseline demographics and study characteristics are highlighted in $>$ Table 1 . The type of vacuum and sponge type are delineated in Supplementary Table 2. The causes of esophageal defects were as follows: surgery $74.0 \%$, nonsurgical iatrogenic $17.5 \%$, and spontaneous $8.5 \%$.

\section{Outcomes}

The outcomes for individual studies are listed in $\mathbf{D}$ Table 2. A total of 17 studies assessed technical and clinical success. The technical success rate was found to be $97.1 \%$ (Cl 95.4\%-98.7\%, PI: $95.2 \%-98.9 \%, l^{2}=0 \%$ ) ( $\mathbf{F i g . 2 a}$ ). The clinical success rate
347 records identified through database

searching

- 5 in Cochrane Library

- 141 in Embase

- 76 in Pubmed/Medline

- 125 in Web of Science

- 119 in Google Scholar

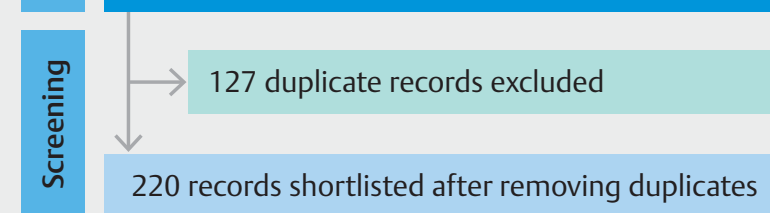

220 records shortlisted after removing duplicates
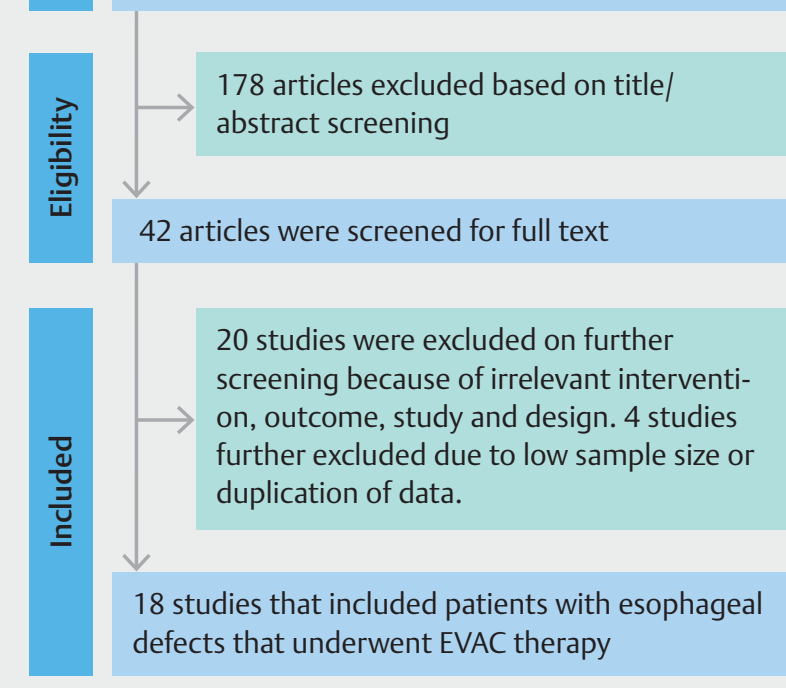

- Fig. 1 PRISMA flow diagram.

was noted to be $89.4 \%\left(\mathrm{Cl} 85.6 \%-93.1 \%, \mathrm{PI}: 80.5 \%-99.3 \%, \mathrm{P}^{2}=\right.$ $36.8 \%$ ) ( Fig. 2b, Supplementary Table 3).

A total of 14 studies assessed the treatment duration, which was found to be 20.3 days ( $\mathrm{Cl} 14.9-25.7, \mathrm{Pl}: 0.0-42.5$, $r^{2}=96.1 \%$ ) ( Fig. 3a). The length of hospital stay was 56.5 days (10 studies, $\mathrm{Cl}$ : 46.6-66.4, PI: 20.1-92.9, $R^{2}=89.5 \%$ ) ( Fig. 3b).

A total 18 studies assessed the overall mortality for patients undergoing EVAC therapy. The rate of mortality was $7.1 \%(\mathrm{Cl}$ $4.7 \%-9.5 \%$, PI: $5.8 \%-10.5 \%, R^{2}=0 \%$ ) ( Fig. 4 a). The overall AE rate for use of EVAC was $13.6 \%$ (Cl $8.0 \%-19.1 \%$, PI: $8.9 \%-$ $20.7 \%, R^{2}=73.6 \%$ ) ( Fig. 4 b). The nature or type of AEs are highlighted in detail in $>$ Table 2 for individual studies. The need for adjuvant or second therapy was $15.7 \%$ (9.8\%-21.6\%, PI: $10.4 \%-23.7 \%, I^{2}=71.3 \%$ ) ( Fig. $4 \mathbf{c}$ ).

\section{Subgroup analysis}

Studies with $\geq 20$ patients

The overall technical success rate was $96.6 \%$ (Cl: $94.3 \%-98.9 \%$, PI: $\left.94.0 \%-99.3 \%, I^{2}=24.4 \%\right)$. The clinical success rate was $88.9 \%$ (Cl: $\left.84.4 \%-93.3 \%, \mathrm{PI}: 81.1 \%-97.5 \%, \mathrm{R}^{2}=38.5 \%\right)$. The overall rates of mortality and AEs were $7.0 \%$ (Cl: $4.2 \%-9.8 \%$, PI: $4.8 \%-$ 
- Table 1 Baseline study characteristics and demographics of included patients.

\begin{tabular}{|c|c|c|c|c|c|c|c|c|}
\hline \multirow[t]{2}{*}{ Study } & \multirow[t]{2}{*}{ Year } & \multirow[t]{2}{*}{ Study type } & \multirow{2}{*}{$\begin{array}{l}\text { Total } \\
\text { patients }\end{array}$} & \multirow{2}{*}{$\begin{array}{l}\text { Mean age, } \\
\text { years (SD) }\end{array}$} & \multirow[t]{2}{*}{ Male, n (\%) } & \multicolumn{3}{|c|}{ Etiology of "defect", $n$} \\
\hline & & & & & & Surgery & $\begin{array}{l}\text { Nonsurgical } \\
\text { iatrogenic }\end{array}$ & $\begin{array}{l}\text { Sponta- } \\
\text { neous }\end{array}$ \\
\hline Berlth et al. & 2018 & Retrospective & 35 & $64.3(11.8)$ & $29(85.3 \%)$ & 35 & 0 & 0 \\
\hline Bludau et al. & 2018 & Retrospective & 77 & $63.2(14.1)$ & $51(66.2 \%)$ & 59 & 12 & 6 \\
\hline Brangewitz et al. & 2013 & Retrospective & 32 & $63.8(11.3)$ & $28(87.5 \%)$ & 30 & 1 & 1 \\
\hline Heits et al. & 2014 & NR & 10 & $66(10.6)$ & $5(50.0 \%)$ & 0 & 4 & 6 \\
\hline Jeon et al. & 2019 & Retrospective & 22 & $67.3(7.2)$ & $17(77.3 \%)$ & 22 & 0 & 0 \\
\hline Jung et al. & 2020 & Retrospective & 30 & 65.4 & $20(66.7 \%)$ & 23 & 5 & 2 \\
\hline Kuehn et al. & 2015 & Retrospective & 21 & $68.3(8.9)$ & $15(71.4 \%)$ & 11 & 8 & 2 \\
\hline Laukoetter et al. & 2016 & Prospective & 52 & $66.3(15.3)$ & $37(71.2 \%)$ & 39 & 9 & 4 \\
\hline Loske (1) et al. & 2011 & Case Series & 14 & NR & NR & 10 & 3 & 1 \\
\hline Loske (2) et al. & 2015 & Case Series & 10 & NR & $7(70.0 \%)$ & 0 & 10 & 0 \\
\hline Mencio et al. & 2018 & Retrospective & 15 & 59.8 & $8(53.3 \%)$ & 2 & 8 & 5 \\
\hline Mennigen et al. & 2015 & Retrospective & 15 & $57.5(9.8)$ & $14(93.3 \%)$ & 15 & 0 & 0 \\
\hline Min et al. & 2019 & Retrospective & 20 & 65.7 (6.9) & $20(100.0 \%)$ & 20 & 0 & 0 \\
\hline Moschler et al. & 2015 & $N R$ & 10 & $73.9(11.7)$ & $5(50 \%)$ & 5 & 4 & 1 \\
\hline Noh et al. & 2018 & Retrospective & 12 & $57.1(3.3)$ & $12(100.0 \%)$ & 12 & 0 & 0 \\
\hline Ooi et al. & 2018 & Retrospective & 10 & $56.7(12.3)$ & NR & 6 & 3 & 1 \\
\hline Pournaras et al. & 2018 & NR & 21 & NR & NR & 7 & 7 & 7 \\
\hline Schneiwind et al. & 2013 & Retrospective & 17 & NR & NR & 17 & 0 & 0 \\
\hline
\end{tabular}

$\left.10.3 \%, R^{2}=0 \%\right)$ and $15.0 \%\left(\mathrm{Cl}: 7.5 \%-22.6 \%, \mathrm{Pl}: 8.9 \%-25.3 \%, R^{2}=\right.$ $78.3 \%)$. The need for adjuvant therapy was $15.8 \%(\mathrm{Cl}: 8.1 \%-$ $23.5 \%$, PI: $\left.9.4 \%-26.6 \%, R^{2}=77.7 \%\right)$. The treatment duration was 24.3 days (7 studies, $\mathrm{Cl}$ : 19.6-29.0 days, Pl: 8.5-40.1 days, $r^{2}=80.6 \%$ ). The length of hospital stay was 54.8 days ( 6 studies, Cl: 40.7-69.0, PI: 7.8-101.8, $\left.I^{2}=92.2 \%\right)$.

\section{Studies published from 2015 on}

The overall technical success rate was $96.9 \%$ (Cl: 95.1\%-98.7\%, PI: $\left.94.9 \%-98.9 \%, R^{2}=39.82 \%\right)$. The clinical success rate was 89.2\% (Cl: 84.9\%-93.6\%, PI: 79.8\%-99.7\%, $P^{2}=46.5 \%$ ). The overall rates of mortality and AEs were $6.6 \%(\mathrm{Cl}: 4.1 \%-9.2 \%$, PI: $\left.4.6 \%-9.6 \%, \mathrm{I}^{2}=0 \%\right)$ and $10.5 \%(\mathrm{Cl}: 5.6 \%-15.4 \%, \mathrm{PI}: 6.7 \%-$ $\left.16.5 \%, R^{2}=62.7 \%\right)$. The need for adjuvant therapy was $17.5 \%$ (Cl: $\left.10.4 \%-24.6 \%, \mathrm{PI}: 11.2 \%-27.4 \%, \mathrm{I}^{2}=77.7 \%\right)$. The treatment duration was 19.9 days ( 11 studies, $\mathrm{Cl}$ : 13.8-25.9 days, PI: 0.0$42.2, r^{2}=96.4 \%$ ). The length of hospital stay was 57.4 days ( 8 studies, $\mathrm{Cl}$ : 43.5-71.2, PI: 8.2-106.6, $\left.\mathrm{I}^{2}=92.6 \%\right)$.

\section{Bias assessment}

The risk of bias for each study using the Newcastle Ottawa Scale is highlighted in Supplementary Table 4. All studies had a score of 6 or more. The inspection of funnel plot revealed visible asymmetry for the studies included on the basis of clinical success with EVAC; however, the overall effect size did not change after adjustments were made using the "trim-and-fill" method (Supplementary Fig. 1). Begg's rank correlation pointed towards a potential publication bias $(P=0.06)$; however, this was not statistically significant.

\section{Discussion}

This systematic review and meta-analysis evaluated the effectiveness of EVAC in the management of esophageal defects. Our results demonstrated high rates of technical and clinical success for EVAC. In addition, rates of mortality and overall AEs associated with the use of EVAC therapy were low. Our results support the potential use of EVAC for management of esophageal defects - leaks, fistula, and perforations of the esophagus. 


\begin{tabular}{|c|c|c|c|c|c|c|c|c|c|c|c|c|c|c|c|c|c|c|c|c|}
\hline \multirow{4}{*}{ 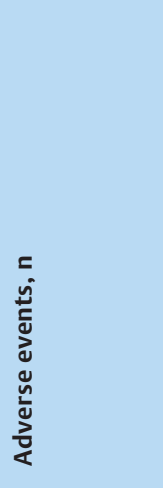 } & 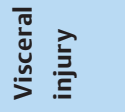 & 0 & 0 & - & $\frac{\tilde{c}}{z}$ & 0 & 0 & 0 & 0 & 0 & 0 & 0 & 0 & 0 & 0 & 0 & $\sim$ & 0 & $\frac{\alpha}{z}$ & \\
\hline & 它 & $\nabla$ & 0 & 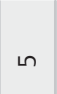 & $\frac{u}{z}$ & 0 & - & 0 & $=$ & 0 & 0 & 0 & 0 & 0 & 0 & 0 & 0 & 0 & $\frac{u}{z}$ & \\
\hline & 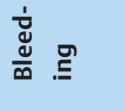 & 0 & $\nabla$ & - & $\frac{\tilde{c}}{z}$ & 0 & $N$ & 0 & in & 0 & 0 & 0 & 0 & 0 & 0 & - & 0 & $\sim$ & $\frac{\mathrm{a}}{z}$ & \\
\hline & 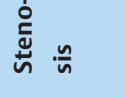 & - & 0 & $m$ & $\frac{\tilde{c}}{z}$ & 0 & 0 & - & $\nabla$ & - & 0 & 0 & 0 & $\wedge$ & - & - & 0 & 0 & $\frac{\alpha^{2}}{2}$ & \\
\hline 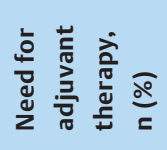 & & 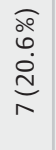 & 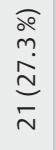 & $\begin{array}{l}\text { ळo } \\
\stackrel{m}{\omega} \\
\stackrel{\omega}{N}\end{array}$ & 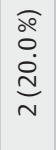 & 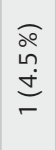 & $\begin{array}{l}\text { ঃ̊ } \\
\stackrel{0}{0} \\
\stackrel{0}{0}\end{array}$ & 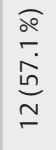 & 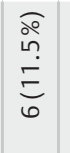 & $\begin{array}{l}\frac{0}{\grave{5}} \\
\frac{5}{5} \\
-\end{array}$ & @̊ & 产 & 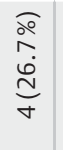 & 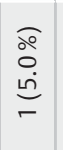 & $\begin{array}{l}\grave{o} \\
\stackrel{0}{0} \\
0 \\
m\end{array}$ & 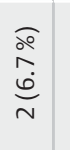 & $\begin{array}{l}\text { ब̊ } \\
0 \\
\dot{0} \\
0 \\
0\end{array}$ & $\begin{array}{l}\text { o̊ } \\
\text { ஸे } \\
\text { ò } \\
\text { N }\end{array}$ & $\frac{\tilde{c}}{z}$ & \\
\hline \multirow{3}{*}{ 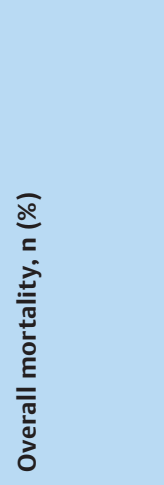 } & 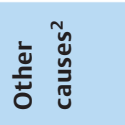 & 㐫 & 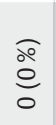 & $\frac{\alpha}{z}$ & $\begin{array}{l}\text { बे } \\
\stackrel{0}{0} \\
\stackrel{0}{-}\end{array}$ & ò & $\begin{array}{l}\text { बo } \\
\stackrel{m}{c} \\
-\end{array}$ & $\frac{\grave{\circ}}{\stackrel{0}{0}}$ & 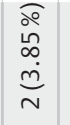 & $\begin{array}{l}\bar{d} \\
\stackrel{5}{5} \\
-\end{array}$ & 㐫 & 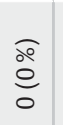 & 仓̊ & 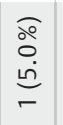 & ঠे & ఏ̊ & 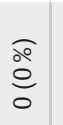 & 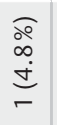 & $\frac{\alpha}{z}$ & \\
\hline & 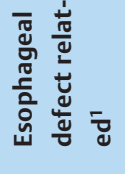 & $\begin{array}{l}\text { ๖o } \\
\grave{b} \\
\stackrel{\infty}{m}\end{array}$ & $\begin{array}{l}\text { oo } \\
\circ \\
\dot{m} \\
\stackrel{0}{0} \\
\stackrel{0}{0}\end{array}$ & $\frac{\mathscr{\alpha}}{z}$ & $\stackrel{\frac{\widehat{o}}{0}}{\stackrel{0}{0}}$ & 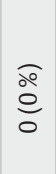 & $\begin{array}{l}\text { बo } \\
\stackrel{m}{m} \\
-\end{array}$ & $\begin{array}{l}\text { ১o } \\
\infty \\
\dot{ \pm} \\
-\end{array}$ & 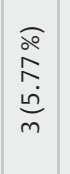 & 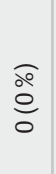 & 离 & 六 & 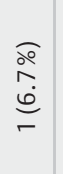 & 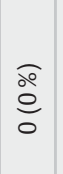 & $\begin{array}{l}\text { ১̊ } \\
0 \\
0 \\
0 \\
m\end{array}$ & 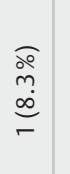 & $\begin{array}{l}\text { ळे } \\
0 \\
\stackrel{0}{0} \\
m\end{array}$ & 仓̊ & $\frac{\alpha}{z}$ & \\
\hline & $\begin{array}{l}\overline{\widetilde{J}} \\
\stackrel{0}{\circ}\end{array}$ & $\begin{array}{l}\text { o̊ } \\
\grave{0} \\
\stackrel{\infty}{m} \\
m\end{array}$ & 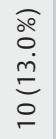 & 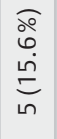 & $\begin{array}{l}\stackrel{\circ}{\circ} \\
\stackrel{0}{\circ} \\
\stackrel{-}{-}\end{array}$ & बे & 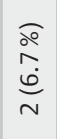 & $\begin{array}{l}\text { ১o } \\
\infty \\
\dot{ \pm} \\
- \\
-\end{array}$ & 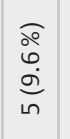 & 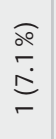 & 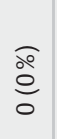 & 仓̊ & 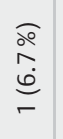 & 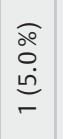 & $\begin{array}{l}\text { ১̊ } \\
\stackrel{0}{0} \\
0 \\
m\end{array}$ & 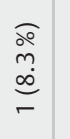 & $\begin{array}{l}\text { ळo } \\
\stackrel{0}{0} \\
\text { m } \\
m\end{array}$ & 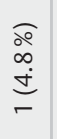 & 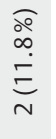 & \\
\hline 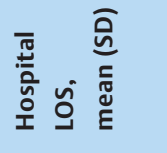 & & 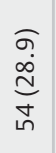 & $\frac{\tilde{c}}{z}$ & 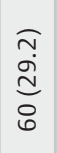 & $\underset{\substack{n \\
\stackrel{\infty}{\sigma}}}{\stackrel{n}{\sigma}}$ & $\begin{array}{l}a \\
m \\
m \\
o \\
m \\
m\end{array}$ & $\frac{\alpha}{z}$ & $\frac{\text { p }}{z}$ & 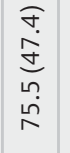 & $\frac{o}{z}$ & $\frac{\tilde{c}}{z}$ & $\frac{a}{2}$ & 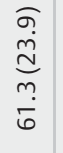 & $\begin{array}{l}\sqrt[n]{n} \\
\stackrel{d}{d} \\
\\
\text { in }\end{array}$ & $\frac{\tilde{c}}{z}$ & $\frac{\alpha}{z}$ & $\begin{array}{l}\widehat{T} \\
\stackrel{1}{E} \\
\infty \\
\dot{P} \\
\stackrel{1}{n}\end{array}$ & $\begin{array}{l}\frac{m}{r} \\
\hat{m} \\
m \\
\frac{m}{b}\end{array}$ & $\begin{array}{l}\widehat{o} \\
\text { in } \\
\text { in }\end{array}$ & \\
\hline 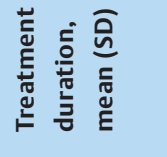 & & 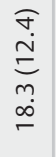 & \begin{tabular}{l}
$\sqrt{n}$ \\
$\infty$ \\
$\stackrel{\infty}{\pi}$ \\
\multirow{N}{N}{}
\end{tabular} & $\begin{array}{l}\underset{m}{\stackrel{n}{d}} \\
\stackrel{m}{m} \\
m \\
m\end{array}$ & $\begin{array}{l}\widehat{m} \\
\stackrel{5}{5} \\
\stackrel{\sigma}{\sigma}\end{array}$ & 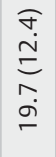 & $\frac{\alpha}{z}$ & 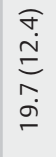 & 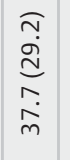 & 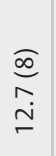 & 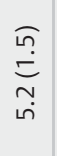 & $\frac{\infty}{2}$ & 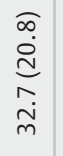 & 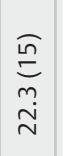 & $\begin{array}{l}\sigma \\
\infty \\
\infty \\
\bar{\sigma} \\
\sigma\end{array}$ & $\begin{array}{l}\frac{\widehat{n}}{\sigma} \\
\stackrel{+}{=}\end{array}$ & 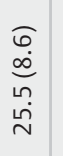 & $\frac{\alpha}{z}$ & $\frac{\alpha}{z}$ & 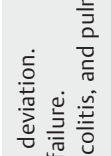 \\
\hline 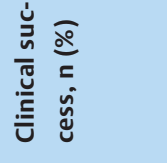 & & 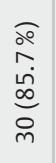 & $\begin{array}{l}\text { ळo } \\
\stackrel{5}{5} \\
\stackrel{5}{\circ}\end{array}$ & $\begin{array}{l}\bar{o} \\
\stackrel{+}{+} \\
\dot{\infty} \\
\stackrel{n}{\infty}\end{array}$ & 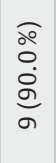 & 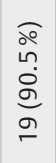 & $\begin{array}{l}\stackrel{\infty}{\infty} \\
m \\
\infty \\
\stackrel{\infty}{n}\end{array}$ & 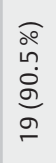 & $\begin{array}{l}\widehat{d} \\
\stackrel{0}{1} \\
\stackrel{d}{0} \\
\text { gे }\end{array}$ & 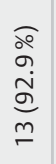 & $\begin{array}{l}\text { o̊ } \\
0 \\
\stackrel{0}{0} \\
\stackrel{0}{\circ}\end{array}$ & $\begin{array}{l}\text { o̊ } \\
0 \\
\dot{0} \\
\stackrel{0}{0} \\
\stackrel{n}{L}\end{array}$ & 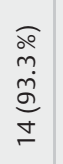 & 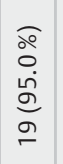 & $\begin{array}{l}\text { ১อ } \\
\vdots \\
\vdots \\
\vdots \\
\wedge\end{array}$ & 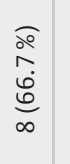 & $\begin{array}{l}\text { बे } \\
0 \\
0 \\
0 \\
0\end{array}$ & 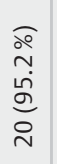 & $\frac{\alpha}{z}$ & 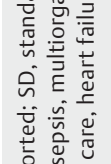 \\
\hline 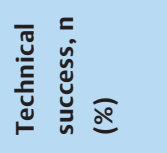 & & 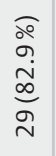 & 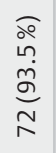 & 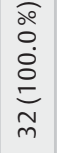 & $\begin{array}{l}\text { कo } \\
\circ \\
0 \\
0 \\
\sigma\end{array}$ & 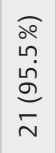 & 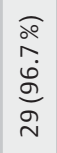 & 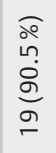 & $\begin{array}{l}\overline{0} \\
0 \\
0 \\
0 \\
0 \\
n \\
n\end{array}$ & 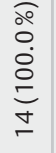 & 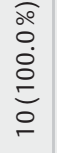 & 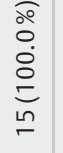 & 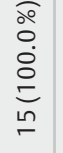 & 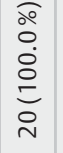 & $\begin{array}{l}\text { ১̊ } \\
0 \\
\stackrel{0}{0} \\
a\end{array}$ & 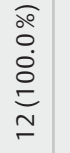 & $\begin{array}{l}\text { ১̊ } \\
\stackrel{0}{0} \\
\stackrel{0}{\sigma}\end{array}$ & 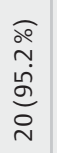 & $\frac{\tilde{u}}{z}$ & 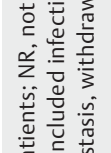 \\
\hline 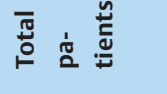 & & $\stackrel{n}{m}$ & 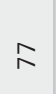 & $\tilde{m}$ & $\stackrel{\circ}{\circ}$ & $\approx$ & 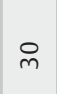 & $\bar{\sim}$ & กี & $\Xi$ & $\stackrel{\circ}{\circ}$ & $\stackrel{\llcorner}{\sim}$ & $\stackrel{\curvearrowleft}{\sim}$ & กิ & 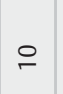 & $\simeq$ & $\stackrel{\circ}{\circ}$ & $\bar{\sim}$ & $=$ & 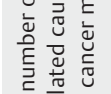 \\
\hline 空 & & 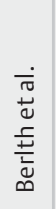 & 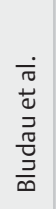 & 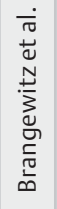 & 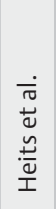 & $\begin{array}{l}\dot{\vec{\pi}} \\
\overrightarrow{0} \\
\overline{0} \\
\stackrel{0}{\underline{0}}\end{array}$ & $\begin{array}{l}\overline{0} \\
\overrightarrow{0} \\
\underline{0} \\
\underline{\Xi}\end{array}$ & 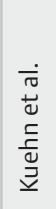 & 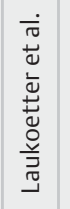 & 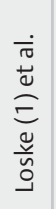 & 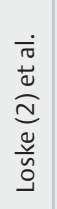 & $\begin{array}{l}\dot{\vec{T}} \\
\overrightarrow{0} \\
\stackrel{0}{\breve{V}} \\
\dot{\tilde{v}} \\
\dot{\Sigma}\end{array}$ & 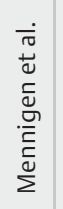 & 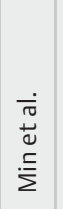 & 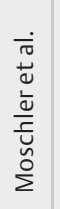 & $\begin{array}{l}\dot{\overrightarrow{0}} \\
\stackrel{0}{0} \\
\frac{1}{0} \\
\dot{2}\end{array}$ & $\begin{array}{l}\dot{\vec{\pi}} \\
\stackrel{ \pm}{ \pm} \\
\dot{0}\end{array}$ & 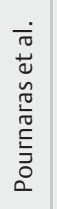 & 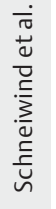 & 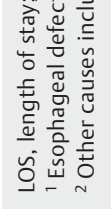 \\
\hline
\end{tabular}




\section{Studies}

Ooi et al. 2018

Berlth et al. 2018

Bludau et al. 2018

Brangewitz et al. 2013

Jeon et al. 2019

Jung et al. 2020

Kuehn et al. 2015

Laukoetter et al. 2016

Loske (1) et al. 2011

Loske (2) et al. 2015

Mencio et al. 2018

Mennigen et al. 2015

Moschler et al. 2015

Noh et al. 2018

Heits et al. 2014

Pournaras et al. 2018

Min et al. 2019

Overall $\left(I^{2}=0 \%, P=0.714\right)$

a

\section{Studies}

\section{Ooi et al. 2018}

Berlth et al. 2018

Bludau et al. 2018

Brangewitz et al. 2013

Jeon et al. 2019

Jung et al. 2020

Kuehn et al. 2015

Laukoetter et al. 2016

Loske (1) et al. 2011

Loske (2) et al. 2015

Mencio et al. 2018

Mennigen et al. 2015

Moschler et al. 2015

Noh et al. 2018

Heits et al. 2014

Pournaras et al. 2018

Min et al. 2019

\section{Estimate (95\% C. I.) $\quad$ Ev/Trt}

$\begin{array}{lr}0.900(0.714,1.000) & 9 / 10 \\ 0.829(0.704,0.953) & 29 / 35 \\ 0.935(0.880,0.990) & 72 / 77 \\ 0.985(0.943,1.000) & 32 / 32 \\ 0.955(0.868,1.000) & 21 / 22 \\ 0.967(0.902,1.000) & 29 / 30 \\ 0.905(0.779,1.000) & 19 / 21 \\ 0.991(0.965,1.000) & 52 / 52 \\ 0.967(0.876,1.000) & 14 / 14 \\ 0.955(0.831,1.000) & 10 / 10 \\ 0.969(0.883,1.000) & 15 / 15 \\ 0.969(0.883,1.000) & 15 / 15 \\ 0.900(0.714,1.000) & 9 / 10 \\ 0.962(0.857,1.000) & 12 / 12 \\ 0.900(0.714,1.000) & 9 / 10 \\ 0.952(0.861,1.000) & 20 / 21 \\ 0.976(0.911,1.000) & 20 / 20 \\ 0.971(0.954,0.987) & 387 / 406\end{array}$

0.75

0.8

0.85

Proportion

\section{Estimate (95\% C. I.) $\quad$ Ev/Trt}

$\begin{array}{lr}0.600(0.296,0.904) & 6 / 10 \\ 0.857(0.741,0.973) & 30 / 35 \\ 0.779(0.687,0.872) & 60 / 77 \\ 0.844(0.718,0.970) & 27 / 32 \\ 0.864(0.720,1.000) & 19 / 22 \\ 0.833(0.700,0.967) & 25 / 30 \\ 0.905(0.779,1.000) & 19 / 21 \\ 0.942(0.879,1.000) & 49 / 52 \\ 0.929(0.794,1.000) & 13 / 14 \\ 0.955(0.831,1.000) & 10 / 10 \\ 0.969(0.883,1.000) & 15 / 15 \\ 0.933(0.807,1.000) & 14 / 15 \\ 0.700(0.416,0.984) & 7 / 10 \\ 0.667(0.400,0.933) & 8 / 12 \\ 0.900(0.714,1.000) & 9 / 10 \\ 0.952(0.861,1.000) & 20 / 21 \\ 0.920(0.854,1.000) & 19 / 20\end{array}$

Overall $\left(I^{2}=36.78 \%, P=0.065\right) \quad 0.894(0.856,0.931) \quad 350 / 406$

b

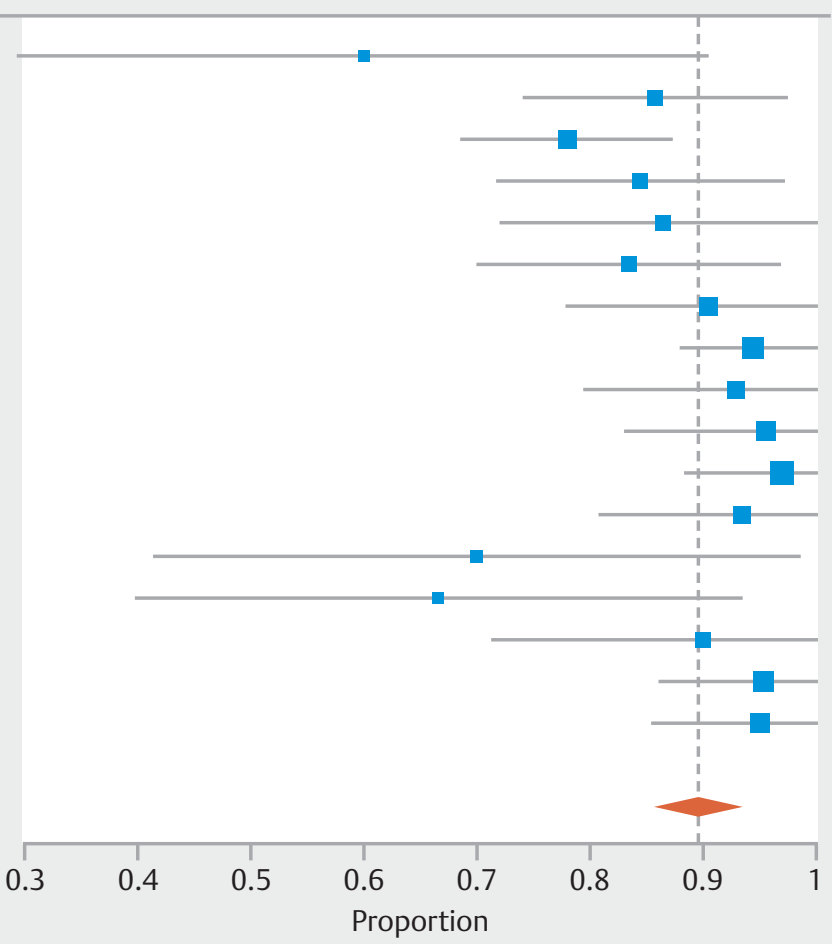

Fig. 2 Forest plot. a Technical success. b Clinical success of EVAC. Cl, confidence interval.

As newer modalities are being assessed for safety and effectiveness, guidelines to treat esophageal transmural defects are evolving [18]. The basic principle is to seal the defect and drain the fluid collection to reduce chance of abscess formation and sepsis. The current approach includes conservative management, surgery, and endoscopy. The conservative approach con- 
Studies

Ooi et al. 2018

Berlth et al. 2018

Bludau et al. 2018

Brangewitz et al. 2013

Jeon et al. 2019

Kuehn et al. 2015

Laukoetter et al. 2016

Loske (1) et al. 2011

Loske (2) et al. 2015

Mennigen et al. 2015

Moschler et al. 2015

Noh et al. 2018

Heits et al. 2014

Min et al. 2019

Overall $\left(I^{2}=96.07 \%, P<0.001\right)$
Estimate (95\% C. I.)

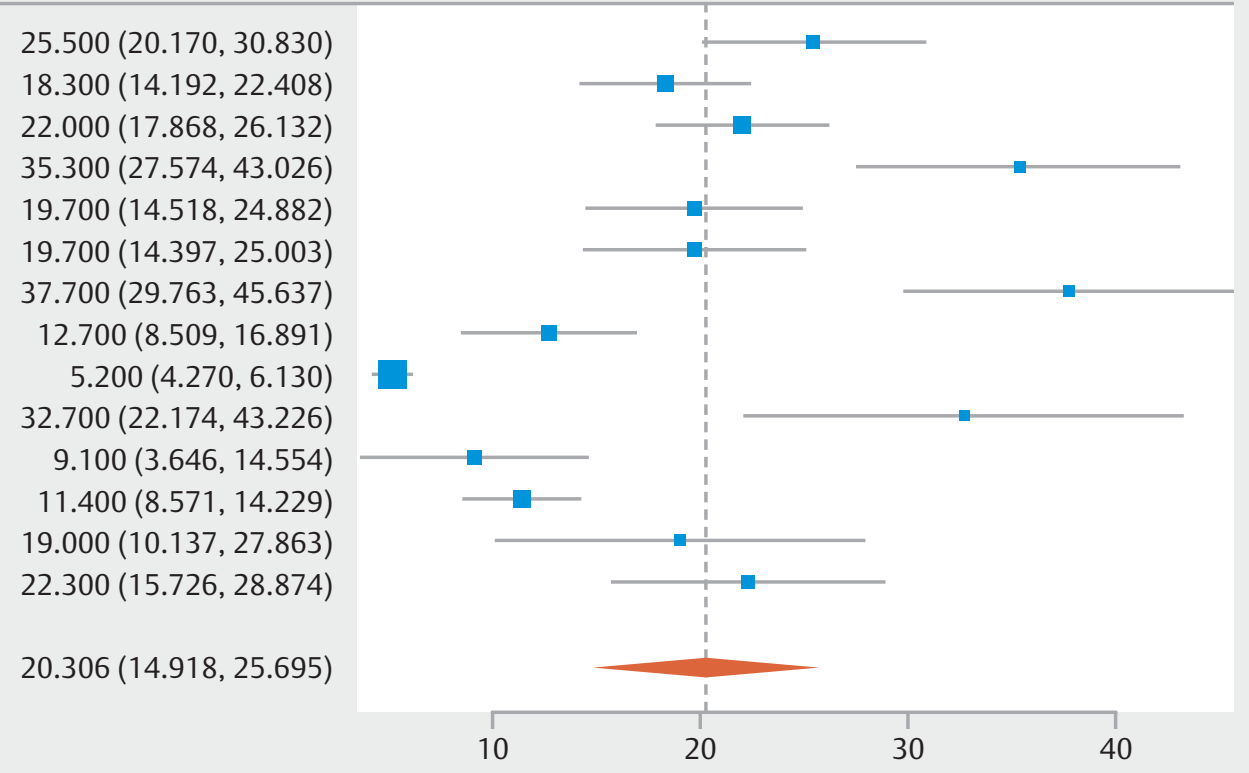

Studies

Estimate (95\% C. I.)

Ooi et al. 2018

Berlth et al. 2018

Brangewitz et al. 2013

Jeon et al. 2019

Laukoetter et al. 2016

Mennigen et al. 2015

Heits et al. 2014

Pournaras et al. 2018

Min et al. 2019

Schneiwind et al. 2013

Overall $\left(I^{2}=89.55 \%, P<0.001\right)$

$56.503(46.584,66.421)$

b

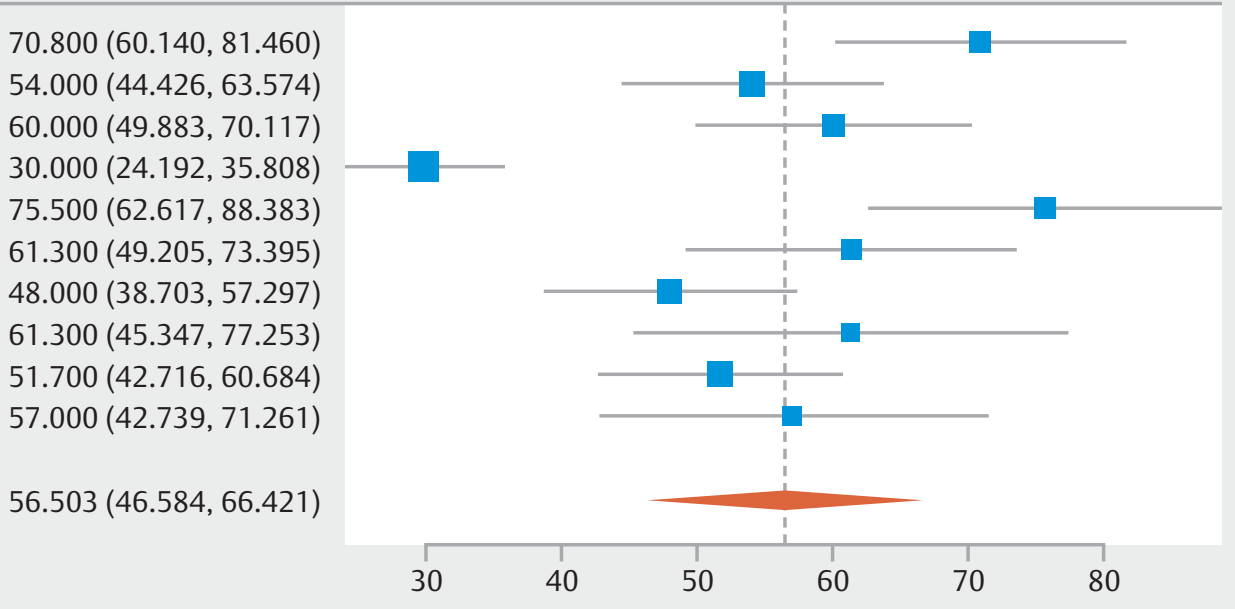

- Fig. 3 Forest plot. a Treatment duration. b Length of hospital stay. Cl, confidence interval.

sists of antibiotic therapy, parenteral nutrition, and insertion of nasogastric tube placement under direct vision [18]. A conservative approach is used for small leaks with early diagnosis and no sepsis. Surgery is indicated in patients with large leaks, significant necrosis, leaks unresponsive to other modalities, and those who have sepsis $[19,20]$. Surgical approaches include direct suturing, enforced suturing, pleural patches, or flaps which are reserved for more severe cases. Endoscopic approaches using clips, stents, suturing, or sealants are an alternative option for cases between the two extremes of the severity spectrum [21]. The clips can be either through-the-scope clips (TTSC) or over-the-scope clips (OTSC). For clip-based closure, the tissue surrounding the clip must be healthy, allowing adherence of clip. Friable, necrotic, or weak tissue will result in technical and clinical failure. The use of TTSC is limited to sealing very small defects, owing to their size. In a recent pooled analysis, OTSC was noted to achieve a clinical success rate of $78.4 \%$ [22]. Esophageal stents, particularly self-expandable stents, are more popular for endoscopic management of leaks, fistula, and perforation. A recent systematic review demonstrated the overall clinical success of self-expandable stents to be $76.8 \%$ [23]. In addition, stents are associated with AEs including fistulas, bleeding, migration, perforation, necrosis, and stricture [24]. Although a direct comparison was not feasible, we demonstrated a clinical success rate of $89.3 \%$ for EVAC in successful closure of esophageal defects, which is notably higher than for either OTSC or stents.

In recent meta-analyses, EVAC was compared to SEMS and a lower overall AE rate (risk difference: $0.24,95 \% \mathrm{Cl} 0.13-0.35$ ) and mortality (OR: $0.39,95 \% \mathrm{Cl} 0.18-0.83$ ) was noted for 


\section{Studies}

Ooi et al. 2018

Berlth et al. 2018

Bludau et al. 2018

Brangewitz et al. 2013

Jeon et al. 2019

Jung et al. 2020

Kuehn et al. 2015

Laukoetter et al. 2016

Loske (1) et al. 2011

Loske (2) et al. 2015

Mencio et al. 2018

Mennigen et al. 2015

Moschler et al. 2015

Noh et al. 2018

Heits et al. 2014

Pournaras et al. 2018

Min et al. 2019

Schneiwind et al. 2013

Overall $\left(I^{2}=0 \%, P=0.639\right)$

a

\section{Estimate (95\% C. I.) $\quad$ Ev/Trt}

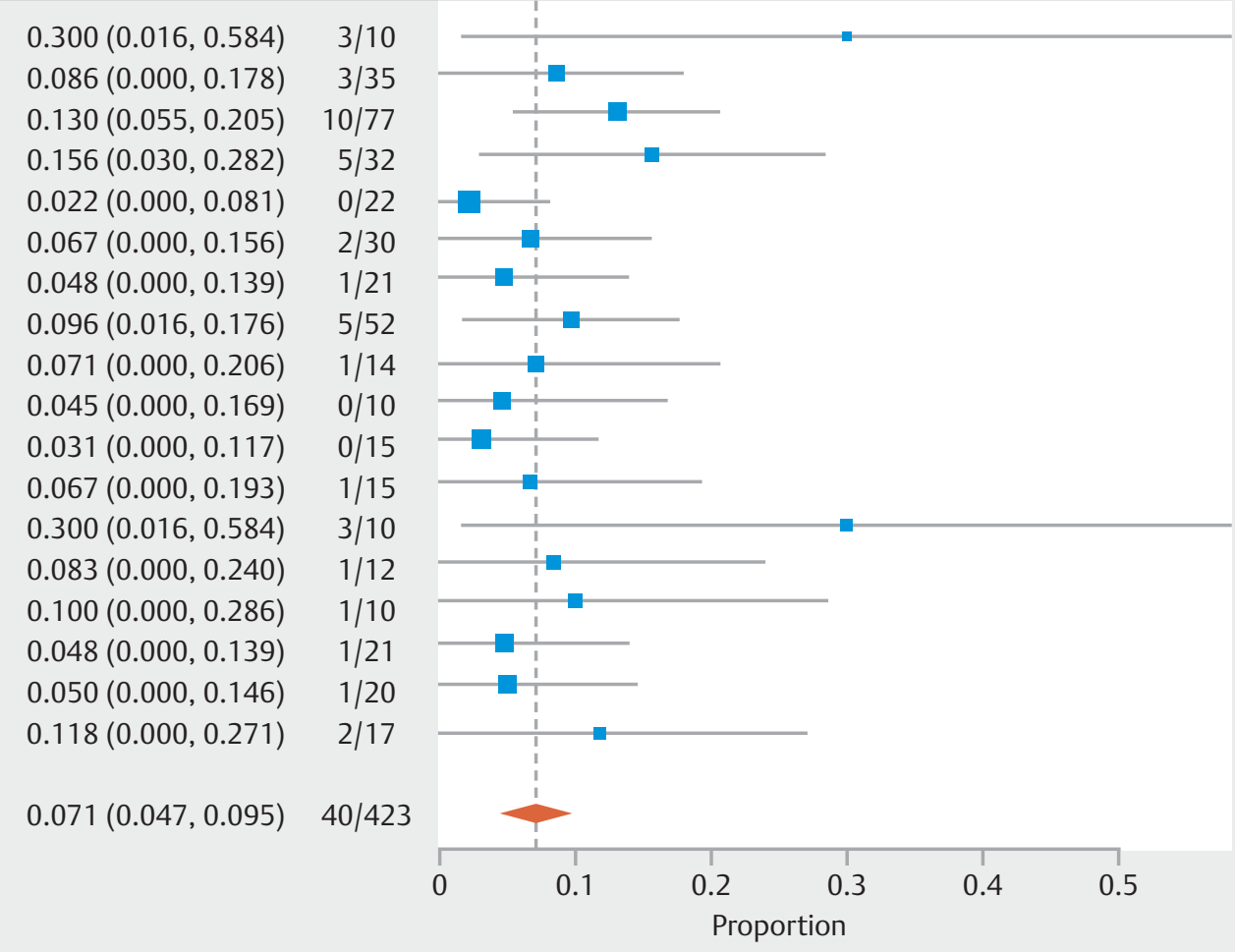

Fig. 4 Forest plot. a Overall mortality.

\section{Studies}

Ooi et al. 2018

Berlth et al. 2018

Bludau et al. 2018

Brangewitz et al. 2013

Jeon et al. 2019

Jung et al. 2020

Kuehn et al. 2015

Laukoetter et al. 2016

Loske (1) et al. 2011

Loske (2) et al. 2015

Mencio et al. 2018

Mennigen et al. 2015

Moschler et al. 2015

Noh et al. 2018

Heits et al. 2014

Pournaras et al. 2018

Min et al. 2019

Overall $\left(I^{2}=73.57 \%, P<0.001\right)$

b

\section{Estimate (95\% C. I.) $\quad$ Ev/Trt}

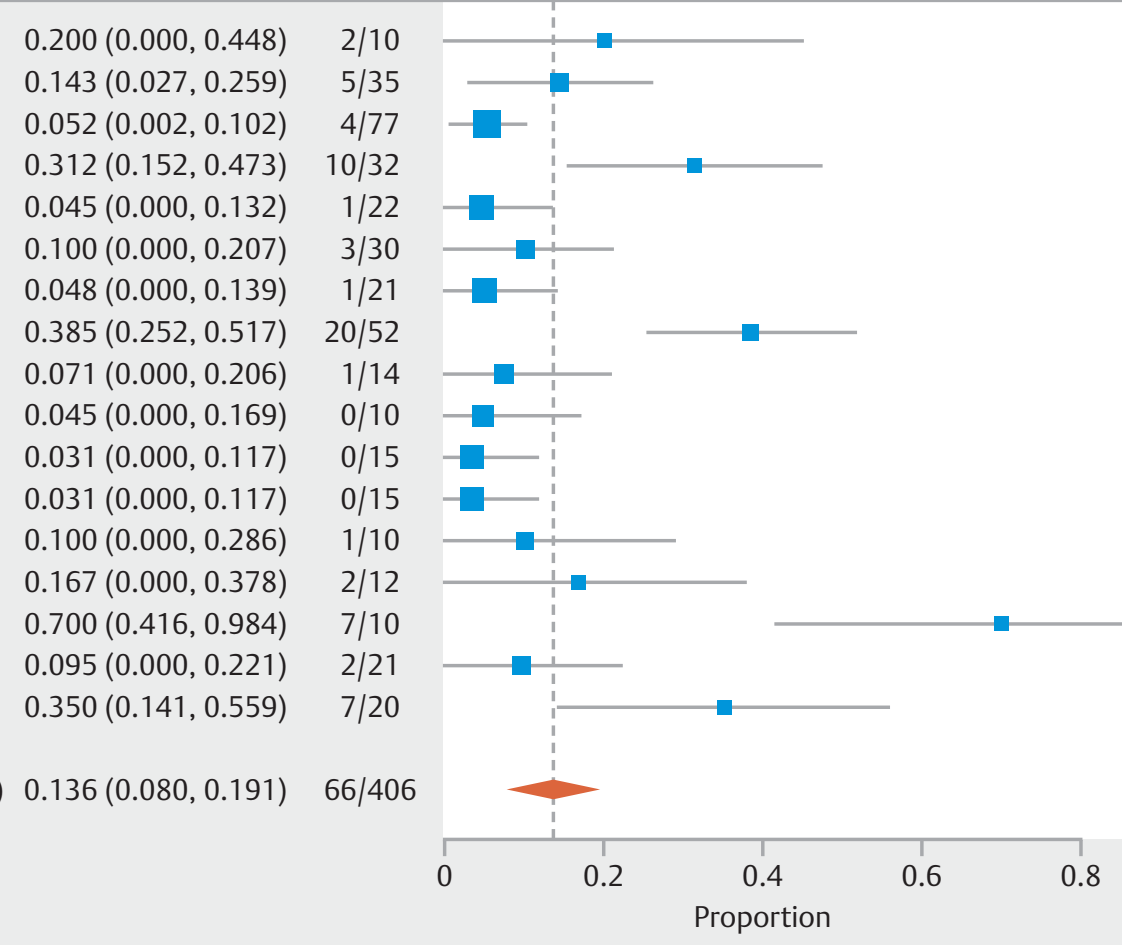

Fig. 4 Forest plot. $\mathbf{b}$ Any adverse events. 


\section{Studies}

Ooi et al. 2018

Berlth et al. 2018

Bludau et al. 2018

Brangewitz et al. 2013

Jeon et al. 2019

Jung et al. 2020

Kuehn et al. 2015

Laukoetter et al. 2016

Loske (1) et al. 2011

Loske (2) et al. 2015

Mencio et al. 2018

Mennigen et al. 2015

Moschler et al. 2015

Noh et al. 2018

Heits et al. 2014

Pournaras et al. 2018

Min et al. 2019

Overall $\left(I^{2}=71.13 \%, P<0.001\right)$
Estimate (95\% C. I.) Ev/Trt

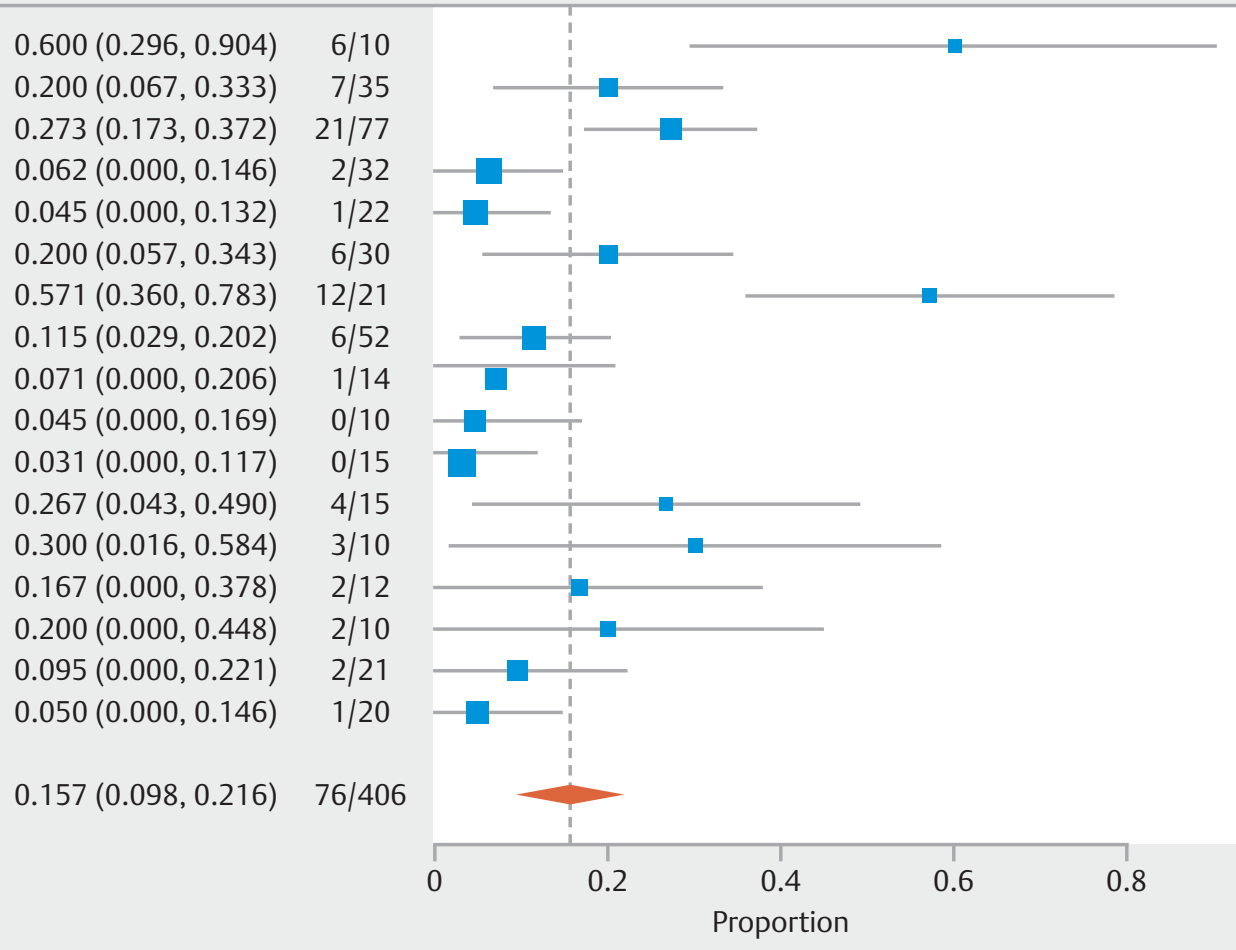

> Fig. 4 Forest plot. c Need for adjuvant therapy. Cl, confidence interval.

EVAC $[25,26]$. The reason for fewer AEs with EVAC may stem from the difference in technique. Because the sponges need replacement every 3 to 4 days, wound healing can be observed every time sponges are exchanged [24]. We speculate that because there are short intervals of wound assessment, the presence of AEs can be detected before progression. This is not feasible with the covered SEMS. Moreover, in case of fluid collection in SEMS, drainage has to be performed externally [27]. Fluid accumulations are drained through a negative pressure apparatus with EVAC, while there is no negative pressure drainage with SEMS. This may contribute to the better safety profile for the EVAC compared with SEMS. Considering the fact that EVAC is relatively novel and there is variability in technique regarding amount of negative pressure and interval of sponge change, the rates of AEs, treatment duration, and length of hospital stay are variable, which may explain the heterogeneity noted in our analysis. As techniques are refined, future AE rates should be even lower than current reports.

Our study had some limitations. The included studies had inherent bias, given their observational nature. Moreover, the patient population was heterogenous. The reasons for that are manifold. First, esophageal transmural defects are secondary to a wide spectrum of etiologies. Furthermore, factors such as site and size of defect, presence of comorbidities, and treatment delay due to difficulty in diagnosis increase the heterogeneity of the patient population. In addition, because the EVAC technique is operator dependent and there have been no clear treatment algorithms such as for the extent of negative pres- sure and interval of sponge change, this would also increase the heterogeneity of cases. EVAC therapy is performed in advanced centers by highly skilled endoscopists, which may affect the reported effectiveness rate. Finally, our study suggests a potential publication bias, with negative studies possibly not being reported. Thus, our results should be interpreted with caution as there may be overestimation in terms of clinical effectiveness and success. Despite the limitations, there are several strengths. Our study is the first to pool the outcomes related to EVAC therapy from a diverse population. We used a stringent search strategy and performed an exhaustive search to include pertinent studies. Our major outcomes - technical success, clinical success, and overall mortality - did not have significant heterogeneity.

\section{Conclusions}

In conclusion, our meta-analysis demonstrated effective outcomes with the use of EVAC for esophageal defects and it should be considered clinically for such patients. Further studies are needed that directly compare EVAC to other modalities, such as OTSC, surgery, and stenting.

\section{Competing interests}

The authors declare that they have no conflict of interest. 


\section{References}

[1] Vidarsdottir $\mathrm{H}$, Blondal S, Alfredsson $\mathrm{H}$ et al. Oesophageal perforations in Iceland: a whole population study on incidence, aetiology and surgical outcome. Thorac Cardiovasc Surg 2010; 58: 476-480

[2] Chirica M, Champault A, Dray X et al. Esophageal perforations. J Visc Surg 2010; 147: e117-e128

[3] Rutegård M, Lagergren P, Rouvelas I et al. Intrathoracic anastomotic leakage and mortality after esophageal cancer resection: a population-based study. Ann Surg Oncol 2012; 19: 99-103

[4] Huu Vinh V, Viet Dang Quang $N$ et al. Surgical management of esophageal perforation: role of primary closure. Asian Cardiovasc Thorac Ann 2019; 27: 192-198

[5] Whyte RI, lannettoni MD, Orringer MB. Intrathoracic esophageal perforation: The merit of primary repair. J Thoracic Cardio Surg 1995; 109: $140-146$

[6] Ong GKB, Freeman RK. Endoscopic management of esophageal leaks. J Thorac Dis 2017; 9: S135-S145

[7] Persson S, Rouvelas I, Irino T et al. Outcomes following the main treatment options in patients with a leaking esophagus: a systematic literature review. Dis Esophagus 2017; 30: 1-10

[8] Hindy P, Hong J, Lam-Tsai Y et al. A comprehensive review of esophageal stents. Gastroenterol Hepatol (NY) 2012; 8: 526-534

[9] Leeds SG, Mencio M, Ontiveros E et al. Endoluminal vacuum therapy: How I do it. J Gastrointest Surg 2019; 23: 1037-1043

[10] Szymanski K, Ontiveros E, Burdick JS et al. Endolumenal vacuum therapy and fistulojejunostomy in the management of sleeve gastrectomy staple line leaks. Case Rep Surg 2018; 2018: 2494069

[11] Kuehn F, Schiffmann L, Janisch F et al. Surgical endoscopic vacuum therapy for defects of the upper gastrointestinal tract. J Gastrointest Surg 2016; 20: 237-243

[12] Moher D, Liberati A, Tetzlaff J et al. Preferred reporting items for systematic reviews and meta-analyses: the PRISMA statement. BMJ 2009; 339: b2535

[13] Stroup DF, Berlin JA, Morton SC et al. Meta-analysis of observational studies in epidemiology: a proposal for reporting. Meta-analysis Of Observational Studies in Epidemiology (MOOSE) group. JAMA 2000; 283: 2008-2012

[14] Goenka MK, Goenka U. Endotherapy of leaks and fistula. World J Gastrointest Endosc 2015; 7: 702-713
[15] Riley RD, Higgins JP, Deeks J]. Interpretation of random effects metaanalyses. BMJ 2011; 342: d549

[16] Higgins J, Thompson SG, Deeks J] et al. Measuring inconsistency in meta-analyses. BMJ 2003; 327: 557-560

[17] Deeks J, Dinnes J, D’Amico R et al. Evaluating non-randomised intervention studies. Health Technol Assess 2003; 7: (iii-x) 1-173

[18] Schaheen L, Blackmon SH, Nason KS. Optimal approach to the management of intrathoracic esophageal leak following esophagectomy: a systematic review. Am J Surg 2014; 208: 536-543

[19] Crestanello JA, Deschamps C, Cassivi SD et al. Selective management of intrathoracic anastomotic leak after esophagectomy. J Thorac Cardiovasc Surg 2005; 129: 254-260

[20] Lampridis S, Mitsos S, Hayward M et al. The insidious presentation and challenging management of esophageal perforation following diagnostic and therapeutic interventions. J Thorac Dis 2020; 12: 27242734

[21] Willingham FF, Buscaglia JM. Endoscopic management of gastrointestinal leaks and fistulae. Clin Gastroenterol Hepatol 2015; 13: 1714-1721

[22] Bartell N, Bittner K, Kaul V et al. Clinical efficacy of the over-the-scope clip device: A systematic review. World J Gastroenterol 2020; 26: 3495-3516

[23] van Halsema EE, van Hooft JE. Clinical outcomes of self-expandable stent placement for benign esophageal diseases: A pooled analysis of the literature. World J Gastrointest Endosc 2015; 7: 135-153

[24] Plum PS, Herbold T, Berlth F et al. Outcome of self-expanding metal stents in the treatment of anastomotic leaks after Ivor Lewis esophagectomy. World J Surg 2019; 43: 862-869

[25] Scognamiglio P, Reeh M, Karstens K et al. Endoscopic vacuum therapy versus stenting for postoperative esophago-enteric anastomotic leakage: systematic review and meta-analysis. Endoscopy 2020; 52: 632-642

[26] Do Monte Junior ES, de Moura DTH, Ribeiro IB et al. Endoscopic vacuum therapy versus endoscopic stenting for upper gastrointestinal transmural defects: Systematic review and meta-analysis. Digest Endosc 2020: doi:10.1111/den.13813

[27] Schubert D, Scheidbach H, Kuhn R et al. Endoscopic treatment of thoracic esophageal anastomotic leaks by using silicone-covered, self-expanding polyester stents. Gastrointest Endosc 2005; 61: 891896 